12

\title{
ESTRATEGIAS DE EXPLICACIÓN Y ARGUMEN- TACIÓN EN EL DISCURSO DE LA MATEMÁTICA. RECURSOS LINGÜÍSTICOS DE LA ORALIDAD Y LA ESCRITURA EMPLEADOS PARA LA ENSEÑANZA Y EL APRENDIZAJE EN LOS PRIMEROS AÑOS DE CARRERAS DE INGENIERÍA
} EXPLANATION AND ARGUMENTATION STRATEGIES IN THE DISCOURSE OF MATHEMATICS. LINGUISTIC RESOURCES OF ORALITY AND WRITING USED FOR TEACHING AND LEARNING IN THE FIRST YEARS OF ENGINEERING CAREERS

Estela Mattioli

mattioli.estela@gmail.com / Profesora adjunta ordinaria e investigadora de la Facultad de Ingeniería y Ciencias Hídricas de la Universidad Nacional del Litoral. Docente en las carreras de Ingeniería Informática, Ambiental, Recursos Hídricos y Agrimensura. Responsable del Taller de Tesis de la Maestría en Ingeniería de los Recursos Hídricos de FICH-UNL. 


\section{RESUMEN}

En este proyecto se recuperarán los resultados que el equipo de trabajo ha alcanzado en experiencias investigativas anteriores, las cuales han abordado las características del discurso de la ciencia en diferentes disciplinas y los modos más productivos de comunicarlo a los estudiantes universitarios para colaborar en su interpretación y reelaboración, promoviendo la construcción de los conocimientos científico-disciplinares específicos. En esta oportunidad se analizarán las estrategias de explicación y argumentación en el discurso de la matemática, y los recursos lingüísticos de la oralidad y la escritura empleados para la enseñanza y el aprendizaje en los primeros años de carreras de ingeniería en el ámbito de la Facultad de Ingeniería y Ciencias Hídricas de la Universidad Nacional del Litoral. Este trabajo interdisciplinario entre docentes de Lengua y Matemática busca describir los modos empleados por expertos y legos para explicar y argumentar, identificando los aspectos que presentan mayor dificultad tanto en el discurso oral como escrito, mediado o no por las TIC, para posteriormente elaborar propuestas de enseñanza a desarrollar dentro de cada asignatura (Comunicación Oral y Escrita y las seis Matemáticas) que favorezcan el desempeño de los estudiantes. El trabajo se enmarcará en la perspectiva que ofrece la Lingüística Sistémico Funcional, además de considerar los aportes del campo de las didácticas específicas y las posibilidades del uso de las nuevas tecnologías de la información y la comunicación (TIC).

\section{PALABRAS CLAVE}

$>$ discurso

$>$ matemática

$>$ argumentación 


\section{ABSTRACT}

This article gives an account about a research which defines its object on the Universidad Nacional del Litoral, based on its relations with the local and socio-political sphere. This relationship expresses an instituting link in a double sense: society challenges university institution and it, in turn, contributes to the constitution of society of which it is a part. This perspective allows to identify and investigate the intersection areas between academic production and cultural and political intervention, considering as well the complex relationship between university and the changing political scenarios of the 20th century in Argentina. In order to develop the research, contributions from renewd political history, history of concepts, intelectual history and discourse analysis are recovered.

This project will recover the results that the work team has achieved in previous research experiences, which have addressed characteristics of science discourse in different disciplines and the most productive ways of communicating it to university students for the purpose of collaborate in its interpretation and rewriting, promoting the construction of specific scientific-disciplinary knowledge. In this opportunity, the explanation and argumentation strategies in the discourse of mathematics will be analyzed, as well as the linguistic resources of orality and writing used for teaching and learning in the first years of engineering careers in the Facultad de Ingeniería y Ciencias Hídricas of Universidad Nacional del Litoral. This interdisciplinary work between Language and Mathematics teachers seeks to describe the ways used by experts and learners to explain and argue, identifying the aspects that present the greatest difficulty both in oral and written discourse, mediated or not by ICT, in order to later elaborate proposals for teaching that will be developed within each subject (Comunicación Oral y Escrita and the six Matemáticas) that promote student's performance. The work will be framed in the perspective offered by Systemic Functional Linguistics, in addition to considering contributions of the specific didactics field and possibilities of using information and communication technologies (ICT).

\section{KEYWORDS}

$>$ discourse

$>$ mathematics

$>$ argumentation 


\section{INTRODUCCIÓN}

En la actualidad las instituciones de educación superior deben hacer frente a una problemática muy compleja como es la deserción y lentificación de los estudiantes en sus carreras de grado, particularmente las pertenecientes a las ingenierías. Dentro de esta complejidad, se puede identificar un aspecto determinante que no siempre es considerado relevante para el desempeño académico en este ámbito: el manejo de las herramientas de lectura y escritura de los textos de ciencia. Estos conocimientos que naturalmente incluyen aspectos conceptuales, actitudinales y procedimentales no pueden dejar de contemplarse como contenidos a trabajar transversalmente en el inicio de los estudios superiores. (Mattioli y Marino, 2012).

Se ha logrado un fuerte consenso sobre la necesidad de que la universidad se haga cargo de la enseñanza de estas prácticas y adherimos a la idea que se constituya en una acción integrada en la que cada profesor incluya dentro del desarrollo específico de contenidos de su área, el trabajo y la reflexión sobre el uso del lenguaje a través del cual es posible aprender ciencia. Alfabetizar académicamente equivale a ayudar a participar en prácticas discursivas contextualizadas que dependen de cada disciplina e implican una formación prolongada que no puede lograrse desde una única asignatura ni en un solo ciclo educativo. Las «alfabetizaciones académicas» incumben a todos los docentes a lo ancho y a lo largo de la universidad (Carlino, 2013: 370).

En el ámbito de las carreras de ingeniería de la Facultad de Ingeniería y Ciencias Hídricas (FICH) de la Universidad Nacional del Litoral (UNL), y más precisamente en el área de matemática, el rendimiento académico del alumno recién ingresado es motivo de permanente análisis y (pre)ocupación por parte de los actores de los procesos de enseñanza y aprendizaje en cuanta instancia de debate educativo se presenta. Desde la comunidad educativa en general, y particularmente desde el equipo docente del área, son incesantes los esfuerzos por revertir el actual estado de situación en este aspecto, sobre el que existe consenso en que dista de ser el esperado, tal como se plasma en los diferentes reportes institucionales de la facultad.

Las estrategias que desde la enseñanza se procuran implementar como aporte para mejorar tal estado de situación, apuntan, entre otras, a dotar y/o reforzar las ausentes o insuficientes competencias cognitivas con las que el estudiante 
da inicio a sus estudios superiores y contribuir a la adquisición de aprendizajes satisfactorios en términos de significación. De allí la conformación de un equipo interdisciplinario de investigación que desde 2016 busca profundizar conocimientos y ganar experticia para el cumplimiento de los fines mencionados. ${ }^{1}$

\section{ESTADO DE LA CUESTIÓN Y MARCO TEÓRICO DE REFERENCIA}

El funcionamiento del lenguaje en contextos específicos ha sido abordado por numerosos autores, los cuales han realizado valiosos aportes a su análisis. Un aporte significativo en la UNL lo constituyen las dos publicaciones de Ghio y Fernández (2005, 2008), quienes abordan desde el enfoque de la Lingüística Sistémico Funcional (LSF) el estudio del español y brindan herramientas para el análisis del discurso y la enseñanza de la lectura y la escritura en diferentes contextos y niveles educativos.

Al mismo tiempo, no puede dejar de considerar el cambio que significa en el aprendiz la transición del pensamiento elemental, propio de la matemática de los niveles anteriores de educación, al avanzado de la universitaria. Diversas investigaciones sobre el tema (Calvo Pesce, 2001, Mariscal Antezana, 2003, entre otras) dan cuenta de las distintas aristas que muestra esta situación, abordando cuestiones cruciales como las vicisitudes del tránsito entre el lenguaje natural y el simbólico y las complicaciones que surgen al enfrentarse por primera vez con la necesidad de argumentaciones y demostraciones rígidas, formales, complejas.

En esta nueva propuesta de trabajo se espera continuar la investigación iniciada en proyectos anteriores sobre el contenido y la organización informativa que presentan los textos de ciencia, fundamentalmente los de matemática, tomando como base la línea teórico-metodológica de la Lingüístico Sistémico Funcional

\footnotetext{
${ }^{1}$ El proyecto CAI+D fue aprobado por la Secretaría de Ciencia, Arte y Tecnología de la UNL en la convocatoria 2020. El grupo responsable está integrado por Estela Mattioli (directora), Mario Garelik (co-director), Analía Demarchi, M. Ofelia Zanetta, Fabiana Montenegro, Elisabet Haye y Silvia Seluy. El grupo colaborador, por Andrés Efron, Evelyn Schalbetter, Laura Gomez, Agostina Zucarelli, María Florencia Acosta, Patricia Schapschuk y Alejandra Gagliardi.
} 
(LSF) iniciada por Michael Halliday (1978), cuyas categorías de análisis permiten identificar los distintos segmentos con diferentes funciones comunicativas dentro de los textos para el intercambio de significados.

Estudios experimentales realizados por D'Andrea et al (2014) durante cuatro años sobre un recorte de la población total de estudiantes de una facultad de ingeniería, mostraron que los ingresantes no se desempeñan adecuadamente en la tarea de la justificación. Ante la premisa de determinar el valor de verdad de ciertas proposiciones, un importante porcentaje de ellos pudo responder correctamente a la consigna pero no sostener o justificar dicha respuesta.

Con respecto al modo de proceder frente a la lectura, Sastre Vázquez et al (2008) clarifican esta cuestión cuando postulan que «una parte importante de las dificultades de los alumnos ante la resolución de problemas matemáticos se debe a no poder dar 'el primer paso', el que consideramos básico y fundamental, que es la lectura comprensiva del enunciado del problema, su interpretación acabada, que es la base sobre la cual deberá construirse la posterior resolución, que también puede presentar problemas, pero de otro tipo.» (p.8.)

Para el Enfoque Ontosemiótico del Conocimiento y la Instrucción Matemática (EOS), comprender un objeto matemático significa organizar la red de relaciones que se deben establecer en la resolución de una situación problemática que obliga al funcionamiento de procedimientos, definiciones, propiedades, argumentos que validan las acciones realizadas, todas ellas soportadas y reguladas por el lenguaje propio de la matemática y el lenguaje natural. En el contexto de alumnos de primer año de universidad que, probablemente, no emplearon libros de texto previamente para estudiar matemática, el armado de las relaciones entre los objetos mencionados (situaciones, procedimientos, definiciones, propiedades, argumentos, lenguaje) puede afectar su comprensión de los contenidos disciplinares (Font y Godino, 2006).

Desde la perspectiva estrictamente lingüística, en la clase de Comunicación Oral y Escrita de FICH, asignatura que se dicta en el primer año de todas las carreras de la facultad, es habitual observar confusión en el uso de ciertos términos como verificar y justificar, así como también resulta confusa la función de justificar respecto de la de explicar, debido en parte a la forma de presentación de los enunciados, los cuales no necesariamente muestran en el nivel superficial del lenguaje (los recursos utilizados) las diferencias de sentido que el hablante 
atribuye a su discurso. Incluso a veces, sólo el contexto puede determinar si el enunciado en cuestión ofrece una explicación o un argumento.

Desde el punto de vista lógico, en una explicación sabemos que algo es verdad y tratamos de arrojar luz sobre las condiciones que lo desencadenaron o hicieron posible, la función comunicativa es mostrar por qué algo es el caso. En una argumentación, en cambio, ofrecemos razones para creer que algo es verdadero y la función comunicativa es mostrar que algo es el caso. Para determinar si una cierta concatenación de afirmaciones constituye un argumento o una explicación, es preciso identificar primeramente la afirmación que hace las veces de conclusión (en general aquella afirmación que precede al término «porque»). Luego si esta afirmación describe algo aceptado como un hecho, el pasaje en cuestión contiene una explicación, en caso contrario estamos frente a una estrategia argumentativa. (Arroyo, 2012: 17).

Como sostienen Godino, Batanero y Font (2004): «Lo que confiere un carácter distintivo al conocimiento matemático es su enorme poder como instrumento de comunicación, conciso y sin ambigüedades» (p. 29). En este marco, el desarrollo de la capacidad para comunicar de forma oral y escrita ese conocimiento resulta fundamental. Investigaciones como la de Jiménez Espinosa, Suárez Ávila y Galindo Mendoza (2010), sostienen que no es sino a través del lenguaje oral como se da soporte al pensamiento y se desarrollan los aspectos esenciales del aprendizaje y la enseñanza de las matemáticas y la comunicación de conceptos de la disciplina, ya sea entre los alumnos o con el profesor. Resulta entonces necesaria, en este contexto, la planificación de actividades didácticas en el aula que involucren una jerarquización de la oralidad como instrumento de aprendizaje.

\section{OBJETIVOS}

\section{GENERAL}

> Caracterizar los discursos de Matemática y de otras disciplinas ingenieriles, focalizando en los aspectos que dificultan la lectura y producción de explicaciones y argumentaciones por parte de los estudiantes que inician sus estudios universitarios. 
$>$ Elaborar propuestas didácticas para implementar en las clases de Lengua y Matemática que brinden herramientas para comprender y producir discursos en las asignaturas de la carrera.

\section{ESPECÍFICOS:}

$>$ Estudiar las relaciones que se establecen entre los significados y el lenguaje analizando las opciones semánticas que éste ofrece y las elecciones que realizan los usuarios para explicar y argumentar dentro de los campos disciplinares de matemática en las carreras de ingeniería.

$>$ Analizar el desarrollo informativo de los textos orales y escritos elaborados por expertos y aprendices que circulan en FICH para las actividades de enseñanza y aprendizaje.

$>$ Identificar las categorías propuestas por la LSF que resultan pertinentes para trabajar en el aula de $\mathrm{COE}$, como herramientas que garanticen la comprensión y producción de textos en la comunidad discursiva de $\mathrm{FICH}$.

$>$ Elaborar propuestas didácticas específicas para cada asignatura, incorporando distintas actividades y recursos innovadores que mejoren la lectocomprensión y elaboración de los textos disciplinares.

\section{ESTRATEGIA METODOLÓGICA}

El marco teórico metodológico es el de la Lingüística Sistémico Funcional (LSF) para el estudio de los discursos disciplinares y el desarrollo informativo que presentan los textos seleccionados de Matemática que resulten representativos para el análisis de las explicaciones y argumentaciones. El proyecto en general se inscribe dentro del paradigma interpretativo, que se centra en la comprensión, en el significado de la palabra y de la acción, en el sentido que se expresa en el lenguaje (Vasilachis de Gialdino, 2006) con las ampliaciones provenientes de la lingüística y de la didáctica de la matemática y la química.

El trabajo se aborda a partir de distintos trayectos, y dentro de cada uno, se focaliza en diferentes aspectos de estudio (como por ej. interpretación de con- 
signas, resolución de problemas, justificaciones, exposiciones orales y escritas, uso de las TICs, entre otros). En función de esto, se seleccionan las categorías teóricas, descriptivas y analíticas correspondientes a cada análisis en particular.

En términos generales, se opta por el método de estudio de casos, sobre muestras de textos reales, no representativos desde el punto de vista cuantitativo y estadístico, sino significativos en tanto ejemplos concretos (actualmente utilizados por las cátedras involucradas). El proceso de análisis considerará tres instancias: (a) descripción, (b) interpretación y (c) explicación; aunque se focalizará fundamentalmente en el nivel descriptivo para demostrar cómo los recursos lingüísticos seleccionados del potencial de significado hacen posible la interpretación y la elaboración de modelos conceptuales explicativos (Rodríguez, Gil y García, 1996). Este modo de análisis permitirá establecer interpretaciones sobre el funcionamiento de las opciones lingüísticas y simbólicas realizadas por los expertos disciplinares en relación con el modo de presentación de la información del campo para hacer comprender un determinado tema o para presentar consignas de trabajo.

Para asegurar un análisis profundo e integrado, se utilizará la estrategia de triangulación metodológica, que consiste en el control cruzado entre diferentes fuentes de datos o la combinación de éstos (Oppermann, 2000). El objetivo será realizar un abordaje multifocal del objeto de investigación que permita extraer del mismo su máximo potencial de significados e indagarlo en el contexto real de su puesta en funcionamiento, combinando diversas técnicas cualitativas (análisis documental, cuestionarios de consignas, entrevistas y observaciones etnográficas) y cuantitativas (tablas y registros numéricos), estos últimos principalmente en la fase de recolección de datos y en una primera etapa del análisis de los mismos.

También se espera aplicar la lingüística contrastiva para el establecimiento de semejanzas y diferencias entre los modos comunicativos de las ciencias «duras» y las sociales, así como detectar y predecir regularidades del género al que pertenecen (Moreno 2008). 


\section{BIBLIOGRAFÍA}

Arroyo G. (2012). Introducción a la lógica y a la argumentación filosófica. Buenos Aires, UNGS.

Ausubel, D. (2002). Adquisición y retención del conocimiento. Una perspectiva cognitiva. Barcelona: Editorial Paidós.

Bazerman et al (2005). Reference guide to Writing Across the Curriculum. West Lafayette, Indiana. The WAC Clearinghouse Parlor Press.

Calvo Pesce, C. (2001). Un estudio sobre el papel de las definiciones y las demostraciones en cursos preuniversitarios de Cálculo Diferencial e Integral (tesis doctoral). Universidad Autónoma de Barcelona. Barcelona. Recuperado de https://ddd.uab.cat/ record/37676.

Cariaga, R. (2014). Grados de hibridación del binomio enseñanza/tecnologías. En Congreso Iberoamericano de Ciencia, Tecnología, Innovación y Educación. Buenos Aires.

Carlino P. (2013). Alfabetización académica diez años después. En Revista Mexicana de Investigación Educativa, vol. 18, núm. 57, 2013, pp. 355-381.

Carmen, M. \& Mejía, L. (2007). «Los métodos en la enseñanza de la matemática. Una experiencia en el contexto histórico-cultural de los alumnos de la carrera de educación básica y de educación media». Acta Latinoamericana de Matemática Educativa Vol.20. pp. 400-405. Fecha de consulta: 7 de marzo de 2020. URLs:http://funes.uniandes.edu.co/5282/1/P\%C3\%A9rezLosm\%C3\%A9todosALME2007.pdf

Castillo, S. y Cabrerizo, J. (2003). Evaluación educativa y promoción escolar. Madrid: Pearson Education.

D'Andrea, R.et al P. (2014). La justificación en estudiantes universitarios de Ingeniería. Congreso Iberoamericano de Ciencia, Tecnología, Innovación y Educación. Buenos Aires, 12 al 14 de setiembre de 2014.

Delgado, A. y Oliver, R. (2006). La evaluación continua en un nuevo escenario docente. Revista de Universidad y Sociedad del Conocimiento. 3(1), p. 2.

Duval, R. (1996). Quel cognitive retenir en didactique des mathématiques? Recherches en Didactique des Mathématiques 6(3), pp. 349-382. file:///C:/Users/eimat/ AppData/Local/Temp/7851-Texto\%20del\%20artículo-22093-2-10-20140805-2.pdf 
Font, V., \& Godino, J.D. (2006). La noción de configuración epistémica como herramienta de análisis de textos matemáticos: su uso en la formación de profesores. Educaçao Matemática Pesquisa, 8 (1), 67-98.

Gee, J.P. (2003). Lo que nos enseñan los videojuegos sobre el aprendizaje y el alfabetismo. Málaga: Ediciones Aljibe.

Ghio E. y Fernández M. (2005). Manual de Lingüística Sistémico Funcional. Aplicaciones a la lengua española. Cuadernos de Cátedra. Santa Fe. Centro de Publicaciones. UNL

Ghio E. y Fernández M. (2008). Lingüística Sistémico Funcional. Aplicaciones a la lengua española. Santa Fe. UNL

Glozman M. y Savio K. (2019). Manual para estudiar textos académicos: prácticas, conceptos y métodos en los procesos de lectura y escritura. Buenos Aires. Noveduc.

Godino, J., Batanero, C. y Font, V. (2004). Didáctica de las Matemáticas para Maestros. manual para el estudiante. Proyecto Edumat-Maestros. ISBN: 84-933517-1-7. URL http://www.ugr.es/local/jgodino/edumat-maestros/. Consultada el 7 de marzo de 2020 Halliday, M.A.K. (1978). El lenguaje como semiótica social. México. Fondo de Cultura Económica.

Jiménez Espinosa, A., Suárez Ávila, Y., Galindo Mendoza, S. (2010). La comunicación: eje en la clase de matemáticas. Praxis \& Saber. Revista de Investigación y Pedagogía. ISSN: 2216-0159. pp. 173-202.

Mariscal Antezana, G. (2003). Una aproximación a la Didáctica en el Proceso del Aprendizaje de las Matemáticas. Disponible en http://www.revistaciencias.com

Martín-Barbero, J. (2003). Saberes hoy: diseminaciones, competencias y transversalidades. Revista Ibero-Americana de Educación. (32), pp. 17-34.

Mattioli E. y Marino F. (2012). Desarrollo de la alfabetización académica en las aulas de ingeniería. Un aporte desde la LSF. Revista de Educación para la Integración. AUGM. Córdoba, UNC

Moreno, A.I. (2008). The importance of comparing comparable corpora in cross-cultural studies. En U. Connor, E. Nagelhout, \& W. Rozycki (eds.). Contrastive rhetoric: Reaching to Intercultural Rhetoric. Ámsterdam.

Oppermann, M. (2000). Triangulation - A Methodological discussion. International Journal of Tourism Research. Vol. 2. N. 2. Pp. 141-146. 
Piaget, J. (1984). La representación en el mundo del niño (6ºdición). Madrid: Ediciones Morata. Revista Educação Matemática Pesquisa, 8(1), 67-98.

Rodriguez G., Gil, J. y García E. (1996). Metodología de la investigación cualitativa. Granada, Aljibe.

Rodríguez Illera, J. (2001). Aprendizaje colaborativo en entornos virtuales. Anuario de Psicología, 32 (2), pp. 63-75.

Rose, D. y Martin, J. (2012). Learning to Write, Reading to Learn: Genre, Knowledge and Pedagogy of the Sydney School. London, Equinox Publishing.

Sáenz Adán, C. (2015). Apoyo del aprendizaje significativo en matemáticas a través de la gamificación (tesis de maestría). Universidad de La Rioja, Logroño, La Rioja. España. Sastre Vázquez, P. et al (2008). La comprensión: proceso lingüístico y matemático. En J. Lezama, M. Sánchez y J. Molina (Eds.) Revista Iberoamericana de Educación. (46), 8-15.

Sepúlveda López, A., Medina García, C. \& Sepúlveda Jáuregui, D. (2009). La resolución de problemas y el uso de tareas en la enseñanza de las matemáticas. Educación matemática. 21(2), 79-115.

Valles, M. (1997). Técnicas cualitativas de investigación social. Madrid. Síntesis.

Vasilachis de Gialdino, I (coord) (2006). Estrategias de Investigación Cualitativa. Barcelona: Gedisa. 